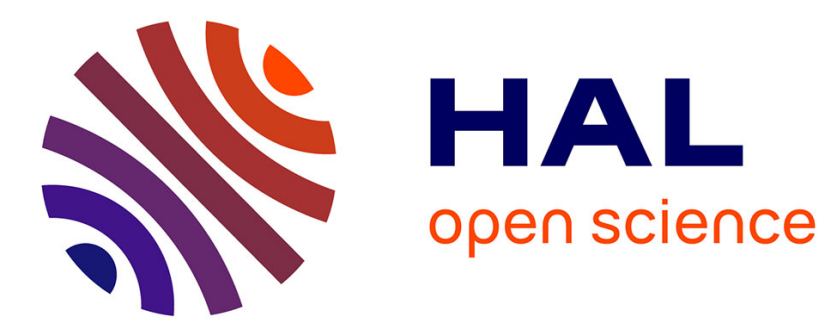

\title{
Strategy for Modeling the Infrared Spectra of Ion-Containing Water Drops
}

Florian Thaunay, Chandramohan Jana, Carine Clavaguéra, Gilles Ohanessian

\section{To cite this version:}

Florian Thaunay, Chandramohan Jana, Carine Clavaguéra, Gilles Ohanessian. Strategy for Modeling the Infrared Spectra of Ion-Containing Water Drops. Journal of Physical Chemistry A, 2018, 122 (3), pp.832-842. 10.1021/acs.jpca.7b10554 . hal-02161555

\section{HAL Id: hal-02161555 \\ https://hal.science/hal-02161555}

Submitted on 21 Jun 2019

HAL is a multi-disciplinary open access archive for the deposit and dissemination of scientific research documents, whether they are published or not. The documents may come from teaching and research institutions in France or abroad, or from public or private research centers.
L'archive ouverte pluridisciplinaire HAL, est destinée au dépôt et à la diffusion de documents scientifiques de niveau recherche, publiés ou non, émanant des établissements d'enseignement et de recherche français ou étrangers, des laboratoires publics ou privés. 


\title{
A Strategy for Modeling the Infrared Spectra of lon-containing Water Drops
}

\author{
Florian Thaunay, ${ }^{\dagger}$ Chandramohan Jana, ${ }^{\dagger}$ Carine Clavaguéra, ${ }^{*} \ddagger$ and Gilles \\ Ohanessian*, \\ $\dagger L C M$, CNRS, Ecole Polytechnique, Université Paris Saclay, 91128 Palaiseau, France \\ $\ddagger$ Laboratoire de Chimie Physique, Université Paris Sud - CNRS, Université Paris Saclay, \\ 15, avenue Jean Perrin, 91405 Orsay Cedex, France \\ E-mail: carine.clavaguera@u-psud.fr; gilles.ohanessian@polytechnique.edu
}




\begin{abstract}
Hydrated ions are ubiquitous in environmental and biological media. Understanding the perturbation exerted by an ion on the water hydrogen bond network is possible in the nano-drop regime by recording vibrational spectra in the $\mathrm{O}-\mathrm{H}$ bond stretching region. This has been achieved experimentally in recent years by forming gaseous ions containing tens to hundreds of water molecules and recording their infrared photodissociation spectra. In this paper, we demonstrate the capabilities of a modelling strategy based on an extension of the AMOEBA polarizable force field to implement water atomic charge fluctuations along with those of intramolecular structure along the dynamics. This supplementary flexibility of non-bonded interactions improves the description of the hydrogen bond network and therefore, the spectroscopic response. Finite temperature IR spectra are obtained from molecular dynamics simulations by computing the Fourier transform of the dipole moment auto-correlation function. Simulations of one to two nanoseconds are required for extensive sampling in order to reproduce the experimental spectra. Furthermore, bands are assigned with the driven molecular dynamics approach. This method package is shown to compare successfully with experimental spectra for 11 ions in water drops containing 36 to 100 water molecules. In particular, band frequency shifts of the free O-H stretching modes at the cluster surface are well reproduced as a function of both ion charge and drop size.
\end{abstract}

\title{
1 Introduction
}

Hydrated ions are ubiquitous in environmental and biological media. The regulation of many metabolic and cellular processes in vivo requires hydrated ions such as $\mathrm{Na}^{+}, \mathrm{K}^{+}, \mathrm{Cl}^{-}$and $\mathrm{SO}_{4}^{2-}$ as nutriments. Their action usually requires partial or total ion dehydration. This has been extensively characterized for signal transduction, which involves crossing membrane channels $^{1,2}$. This principle can also be used to devise ion-selective synthetic nanopores, such as graphene pores ${ }^{3,4}$, with high potential for water filtration and other applications. In the 
atmosphere, several hydrated ions, including sulfate, are present, due in part due to anthropic emissions ${ }^{5}$. They are known to be involved in particle nucleation en route to aerosol particles and cloud condensation nuclei ${ }^{6}$, leading to acidity changes and eventually to acid rains and other climate perturbations. Understanding water's hydrogen bond network and how it is perturbed by the presence of ions is therefore of paramount importance. A large body of results has been obtained on bulk salted water solutions. At the other end of the size spectrum, small ion-water clusters have been extensively considered as they may be generated in a relatively routine way, and because they lend themselves to modelling by highly accurate quantum mechanical methods. There also exists an intermediate regime, in which one ion is surrounded by tens to hundreds of water molecules, which allows studying the transition from molecular to bulk properties. In addition, such nano-drops of growing size can mimic the surface and interface of bulk solutions. Thus this regime may be of great interest. It has only been recently, however, that several research groups have been able to form and structurally characterize such species experimentally ${ }^{7-26}$. Their modelling is also a challenge, and it is the purpose of the present paper to put forth a method to generate infrared (IR) spectra of such species as vibrational spectroscopy has been shown to carry rather useful structural information ${ }^{7-23}$.

Information on the dynamics of hydrated or micro-hydrated ions can be achieved with molecular dynamics (MD) simulations at the DFT level or using classical force fields. With the aim of understanding the structure and the dynamics of large hydrated systems, DFT MD may be restrained to a partial description due to the limitation in the simulation time. Moreover, we will show in this work that the convergence of vibrational spectra requires extensive sampling and therefore a longer simulation time. MD simulations based on classical force fields have been widely used to model ion hydration and have been improved to treat on an equal footing the water-water network and ion-water interactions thanks to the development of polarizable force fields ${ }^{27-33}$. One example is the TTM2-F fluctuating charge 
model developed by Burnham et Xantheas ${ }^{34}$ which was applied to liquid water. The model was able to reproduce the change in the internal geometry of interacting water molecules, as a function of cluster size. Xantheas and Fanourgakis proposed an extension of this model through the TTM3-F potential for which the charge redistribution accounts for the change in the dipole moment due to the change in the geometry of the water molecules ${ }^{35}$. This version was able to reproduce ab initio IR spectra for water clusters with up to 20 water molecules. Indeed, the model is sensitive enough to the environment to yield frequencies in the large range of $3100-3800 \mathrm{~cm}^{-1}$.

We have recently proposed the extension of the use of the AMOEBA (Atomic Multipole Optimized Energetics for Biomolecular Applications) polarizable force field ${ }^{29,36,37}$ to compute finite temperature IR spectra and their mode attributions ${ }^{38,39}$. However, recent work has shown that AMOEBA polarization energies of water clusters are generally too small in comparison to SAPT induction energies ${ }^{40}$. The main reason should come from the lack of charge transfer in the force field model. Some models have been built but heretofore, no obvious way to include these effects and to improve the model has been found ${ }^{40}$. One possibility should be to combine the polarizable model to a partial fluctuation of the atomic charges during the dynamics. The idea is not to replace the polarization term but rather to complement it with an additional contribution. Such model has been implemented in order to study ions included in water drops and to see the sensitivity of the infrared spectra in the $\mathrm{O}-\mathrm{H}$ stretching range.

Herein, we present the implementation of a fluctuating charge model in the AMOEBA14 water model to introduce a part of intramolecular charge transfer into the water molecule. In sections 2 and 3, the new model and the technical details are provided. Then, the computed IR spectra are presented for sulfate-water clusters of various sizes with the aim of reproducing experimental signatures and validating the AMOEBA fluctuating charge model. Mode 
assignments of the IR spectra are proposed in section 4, using the driven molecular dynamics method $^{39}$. Finally, the influence of the ion on the spectra is investigated, following our recent work on the dynamical behaviour of ions confined in a drop of 100 water molecules ${ }^{33}$.

\section{Computational Details}

\subsection{The AMOEBA force field}

The AMOEBA polarizable force field has been shown to be particularly relevant to the computation of chemical properties of hydrated ions including $\mathrm{Na}^{+}, \mathrm{K}^{+}, \mathrm{Cl}^{-29}, \mathrm{Ca}^{2+}, \mathrm{Mg}^{2+41}$, $\mathrm{Zn}^{2+42}, \mathrm{Fe}^{2+43}$, the $\mathrm{Mg}^{2+}$ :phosphate ion pair ${ }^{44}$ or lanthanide and actinide cations ${ }^{45}$. It has the main advantage to combine a complex electrostatic model, including permanent charge, dipole and quadrupole moments on each atom derived from quantum mechanical calculations, and many-body polarization effects which are explicitly treated using a self-consistent atomic dipole polarization procedure ${ }^{37,46}$. Repulsion-dispersion interactions between pairs of nonbonded atoms are represented by a buffered 14-7 potential ${ }^{47}$. The intramolecular valence terms consist in anharmonic bond stretchings and angle bendings, and torsions with the MM3 force field energy form ${ }^{48}$. Additional terms include out-of-plane bending and bondangle cross-terms.

The sulfate parameters were initially taken from previous work ${ }^{49}$. Gas-phase IRMPD spectra of micro-hydrated sulfate clusters $(\mathrm{n}=3-24)$, recorded in the $500-1800 \mathrm{~cm}^{-1}$ range, exhibit a $100 \mathrm{~cm}^{-1}$-wide $\mathrm{S}-\mathrm{O}$ stretch band, centered at $\sim 1100 \mathrm{~cm}^{-150}$. Irrespective of the water model, the AMOEBA band was centered at $1280 \mathrm{~cm}^{-1}$. Accordingly, the bond force constant in the bond stretching term was adjusted from 600 to $400 \mathrm{kcal} . \mathrm{mol}^{-1} . \AA^{-2}$ calibrated on the gas-phase experimental band. It was shown previously that in classical MD simulations, changes in the molecular structures, and even more IR spectra, are very sensitive to the directionality of hydrogen bonds ${ }^{38}$. Based on this previous experience on gas-phase simulations, a set of multipoles was derived for the sulfate dianion, both bare and hydrated by 
six water molecules, using the distributed multipole analysis (DMA) ${ }^{51,52}$ of the MP2/aug-ccpVTZ electron density. Keeping the DMA charges fixed, the atomic dipoles and quadrupoles were then optimized against the electrostatic potential computed at the MP2/aug-cc-pVTZ level. Both sets of sulfate multipoles provide very similar infrared spectra for water drops of different sizes (not shown). Finally, the multipole set extracted for the bare sulfate has been used for this study.

In a recent work, the behavior of various ions carrying a charge from -2 to +3 confined in a drop of 100 water molecules has been followed by MD simulations with AMOEBA ${ }^{33}$. The AMOEBA03 version of the force field has been used and a coherent picture of structural and dynamical properties has been obtained. Recently, a new version of the AMOEBA water model (AMOEBA14) has been proposed thanks to a new parameter calibration procedure ${ }^{53}$. The new model provides a clear improvement in the calculation of bulk water properties, however results for spectroscopic properties were not provided. We have found that the AMOEBA14 version improves significantly the infrared spectra of pure water and sulfatewater drops. To be sure that the structure and dynamics of the ions in water are still valid with the new water model together with unchanged ion parameters, their behavior has been checked by performing MD simulations for various ions in a 100-water molecule drop in similar conditions than in the work described in ref $^{33}$. Ion dynamics as a function of its charge and size is found to be in good overall agreement with the two water models: coordination numbers are well reproduced and the major numerical difference is for ion-water distances in the first coordination shell (an increase for the cations and a decrease for the anions of 5 to $10 \%$ ). Based on these results, all the spectra presented in this study have been obtained using the AMOEBA14 water model.

\subsection{MD simulation and sampling conditions}

MD simulations were carried out for several purposes: (i) providing temperature-equilibrated structures, (ii) exploring potential energy surfaces extensively, (iii) recording dipole moment 
to compute its time autocorrelation function and (iv) exciting atomic motions at specific frequencies to obtain vibrational mode assignments. All simulations have been performed with the TINKER program ${ }^{54}$.

For each size, $\left[\mathrm{SO}_{4}\left(\mathrm{H}_{2} \mathrm{O}\right)_{n}\right]^{2-}$ with $\mathrm{n}=36,55,80,100$, initial structures were generated in a similar way than in our previous work ${ }^{33}$. Then, MD simulations were performed with

a $1 \mathrm{fs}$ time step in the canonical ensemble at $300 \mathrm{~K}$ using the Nosé-Hoover thermostat ${ }^{55}$ for a simulation time of $2 \mathrm{~ns}$, sometimes increased to 3 ns. These conditions allow to reach a correct equilibrium of the structures before the IR spectrum computation.

Relevant starting structures are necessary to perform vibrational mode attributions. Consequently, Replica Exchange Molecular Dynamics (REMD) was used to explore the conformational landscape of the sulfate-water drop with 80 water molecules. REMD simulations were performed using 16 replicas propagated with a time step of $1 \mathrm{fs}$. The temperature range was chosen with a geometric progression between 100 and $350 \mathrm{~K}$ to avoid water evaporation. Exchanges of a random pair of adjacent configurations were attempted every 10 ps and the total simulation time for each replica was $2 \mathrm{~ns}$. At the end, all resulting configurations were optimized with AMOEBA and were sorted to eliminate duplicates.

\subsection{Infrared spectra calculations and mode attributions}

Finite temperature IR spectra were obtained from AMOEBA MD simulations by computing the Fourier transform of the dipole auto-correlation function (DACF-AMOEBA) ${ }^{38}$. First, an equilibration phase is performed during 50 ps using the Nosé-Hoover thermostat. Then, a 200 ps simulation is performed in the microcanonical ensemble using a time step of 0.1 fs, such a small value increasing significantly the length of the simulations. The IR spectra presented in this work are averaged over three to ten such simulations, as indicated.

Band assignment is based on the Driven Molecular-Dynamics (DMD) approach of Bowman and co-workers ${ }^{56-58}$, recently adapted and extended by ourselves ${ }^{39}$. The method consists 
in adding an external driving term in the potential to scan a frequency range and record resonant absorptions. These absorptions can then be related to the vibrational modes of the system. A description of the method is recalled in Supporting Information.

The DMD tool has been applied to provide attributions of the infrared bands of hydrated ions, especially in the $\mathrm{O}-\mathrm{H}$ stretching range. For this size of drop, two to four hydration spheres are populated, resulting in various responses of the $\mathrm{O}-\mathrm{H}$ vibrators due to environment changes along the simulation. The original DMD is useful to obtain attributions but the answer quality may be limited for cases with large density of vibrators. For $n=80$ for example, $160 \mathrm{O}-\mathrm{H}$ vibrators are involved in local or combination modes in the $3100-3750 \mathrm{~cm}^{-1}$ range. In order to provide a full attribution in the $\mathrm{O}-\mathrm{H}$ stretching region, a scan is systematically performed by DMD on the DACF-AMOEBA spectrum without selecting a priori any specific frequencies. For each frequency (using a $1 \mathrm{~cm}^{-1}$ frequency step), a DMD simulation of 0.2 ps is performed and the oscillations are stored for each internal coordinate of the system. Each vibrator is identified and attributed to a molecule from the first, second, third or fourth solvation shell. The method may involve different coupling constants for the driving term due to different environments (see SI). A single value of the coupling constant, $\lambda=2.10^{-7}$ Hartree.Bohr ${ }^{-1}$, was used herein for the entire spectral range to make the method more practical.

\subsection{The AMOEBA fluctuating charge model}

To improve the description of the electrostatic and polarization interactions, atomic charge fluctuation inside each water molecule has been allowed in the AMOEBA force field. This model is derived from the TTM2-F fluctuating charge potential developed by Burnham and Xantheas ${ }^{34}$ for the computation of liquid water properties by improving the ability to adjust intramolecular structural parameters ${ }^{35}$.

Following the original formulation of Xantheas and Fanourgakis ${ }^{35}$, the charge variations 
allowed in the AMOEBA water model are:

$$
\begin{gathered}
\tilde{q}^{H_{1}}=q^{H_{1}}+d_{r}\left(r_{O H_{1}}-r_{e}\right)+d_{\theta}\left(\theta-\theta_{e}\right), \\
\tilde{q}^{H_{2}}=q^{H_{2}}+d_{r}\left(r_{O H_{2}}-r_{e}\right)+d_{\theta}\left(\theta-\theta_{e}\right), \\
\tilde{q}^{O}=-\tilde{q}^{H_{1}}-\tilde{q}^{H_{2}},
\end{gathered}
$$

$\tilde{q}^{H_{1}}$ and $\tilde{q}^{H_{2}}$ are the fluctuating charges derived from the $\mathrm{q}^{H_{1}}$ and $\mathrm{q}^{H_{2}}$ fixed charges, $r_{e}$ and $\theta_{e}$ the equilibrium monomer bond length and angle, respectively. $d_{r}$ and $d_{\theta}$ are parameters that need to be determined. The charge on the oxygen atom is deduced from the hydrogen atom ones to maintain each molecule neutral. We introduce a quadratic variation of the charges as a function of distances and angles in order to impact the geometries far from equilibrium without affecting the free $\mathrm{OH}$ vibrators.

If $\Delta r_{i}=\left(r_{O H_{i}}-r_{e}\right)$ and $\Delta \theta=\left(\theta-\theta_{e}\right)$, the equations can be written as:

$$
\begin{aligned}
& \tilde{q}^{H_{i}}=q^{H_{i}}+0.6\left(1.1 \Delta r_{i}^{2}+0.05 \Delta r_{i}\right)+\left(1.1 \times 10^{-4} \Delta \theta^{2}+5 \times 10^{-4} \Delta \theta\right), \\
& \tilde{q}^{O}=-\tilde{q}^{H_{1}}-\tilde{q}^{H_{2}}
\end{aligned}
$$

The parameters in the equation above have been adjusted in order to reproduce the global shape of the sulfate-water IR spectra for $n=36$ and $n=80$. The fluctuation of the charges is produced by a variation of the intramolecular geometry, consequently, environment effects, such as polarization, are only included indirectly. The impact of the fluctuation of the charges was tested on both geometries and relative energies of water clusters with 20 molecules. It is very limited on the two properties as shown in Table S1 in Supporting Information. 


\section{Results and Discussion}

\subsection{Improvement of the AMOEBA14 water model for IR spec- troscopy}

\subsubsection{IR spectra of water}

As discussed in section 2.1, the AMOEBA14 parameter set has been used for water, together with previously determined parameters for ions. However, there is no previous assessment of these parameters for vibrational spectroscopy. We have therefore carried out calculations of IR spectra for water clusters and sulfate dianion inside water clusters, first comparing AMOEBA14 to AMOEBA03 with water parameters that have been previously modified to match symmetric and antisymmetric O-H stretching frequencies of the bare water molecule ${ }^{37}$. Comparison to experiment indicates that both AMOEBA03 and AMOEBA14 match the three experimental frequencies of isolated $\mathrm{H}_{2} \mathrm{O}$ to within $1 \mathrm{~cm}^{-1}$ (see Table $\mathrm{S} 2$ in Supporting Information). For the water dimer (see Table 1), the DACF-AMOEBA frequencies were calculated at $1 \mathrm{~K}$ to compare to low temperature experiments. An average error of $20 \mathrm{~cm}^{-1}$ is obtained, significantly smaller than with the harmonic frequencies computed at the ab initio MP2/aug-cc-pVTZ level, even when a scaling factor is used, especially for all O-H stretching modes.

When increasing the size of the water cluster, the frequencies that correspond to the bending modes are shifted to the blue in comparison to experiments in a way similar to that found with iAMOEBA ${ }^{62}$ (i.e. inexpensive AMOEBA which uses a simplified polarization calculation), with an overestimate of ca. $100 \mathrm{~cm}^{-1}$ for the value of bulk water. Consequently, two water drops of 21 and 50 molecules have been considered to calibrate the bending force constant in the angle bending function of the force field (see equation 3 in reference ${ }^{53}$ ). The value of $\mathrm{K}_{\theta}=43.65 \mathrm{kcal}_{\mathrm{mol}}{ }^{-1}$.degree ${ }^{-2}$ was used for this study instead of the initial one of 
Table 1: Available experimental vibrational frequencies of the water dimer, their average values, and deviations of unscaled harmonic MP2/aug-cc-pVTZ and DACF-AMOEBA14 values with respect to the experimental average. A and D stand for acceptor and donor molecules, respectively.

\begin{tabular}{lllllll}
\hline $\left.\mathbf{H}_{2} \mathbf{O}\right)_{2}$ & \multicolumn{3}{c}{ Exp. } & & MP2 $^{(c)}$ & AMOEBA14 \\
\hline & $\mathrm{Ar}^{(a)}$ & $\mathrm{N}_{2}{ }^{(a)}$ & $\mathrm{He}^{(b)}$ & Average & apVTZ & DACF-1K \\
A bend & 1593 & 1601 & & 1597 & 27 & 21 \\
$\mathrm{D}$ bend & 1611 & 1619 & & 1615 & 40 & 27 \\
$\mathrm{D}_{\text {sym }}$ stretch & 3574 & 3550 & 3545 & 3556 & 192 & 12 \\
$\mathrm{~A}_{\text {sym }}$ stretch & 3634 & 3627 & 3600 & 3620 & 218 & 20 \\
$\mathrm{D}_{\text {antisym }}$ stretch & 3709 & 3699 & 3714 & 3707 & 227 & 20 \\
$\mathrm{~A}_{\text {antisym }}$ stretch & 3726 & 3715 & 3730 & 3724 & 231 & 11 \\
\hline
\end{tabular}

(a) reference ${ }^{59}$, (b) reference ${ }^{60}$, (c) reference ${ }^{61}$

$48.98 \mathrm{kcal} . \mathrm{mol}^{-1}$.degree ${ }^{-2}$, with a negligible impact on the O-H stretching modes.

\subsubsection{IR spectra of sulfate-water clusters}

The AMOEBA14 model The AMOEBA force field and its standard electrostatic term have been used to compute infrared spectra of the sulfate ion included in water drops with 36, 55, 80 and 100 molecules. The spectra are reported in Figure 1 at temperatures of 100 and $200 \mathrm{~K}$. For the smallest size, the spectrum turns out to be more dependent upon the starting structure of the dynamics than for larger sizes. To bypass this issue, the spectra are averaged over simulations started from four different initial structures, each spectrum of these structures being cumulated over three different simulations of 200 ps. At both temperatures, free $\mathrm{O}-\mathrm{H}$ bands appear only occasionally for $\mathrm{n}=36$ and the intensity of the band increases with the size of the drop, with an overall small red-shift of ca. $20 \mathrm{~cm}^{-1}$ from experiments $^{11}$. Focusing on the bonded $\mathrm{O}-\mathrm{H}$ stretching region, a broad band of ca. $300 \mathrm{~cm}^{-1}$ width is observed centered near $3450 \mathrm{~cm}^{-1}$ and red-shifted with increasing size. However, the spectra in this region show two limitations, (i) the band is less wide than experiments ${ }^{11}$, (ii) the double-band shape is not satisfactory. Several modifications of the sulfate parameters, especially the multipoles, have been attempted. The results emphasize that the problems arise mainly from the water model itself. 

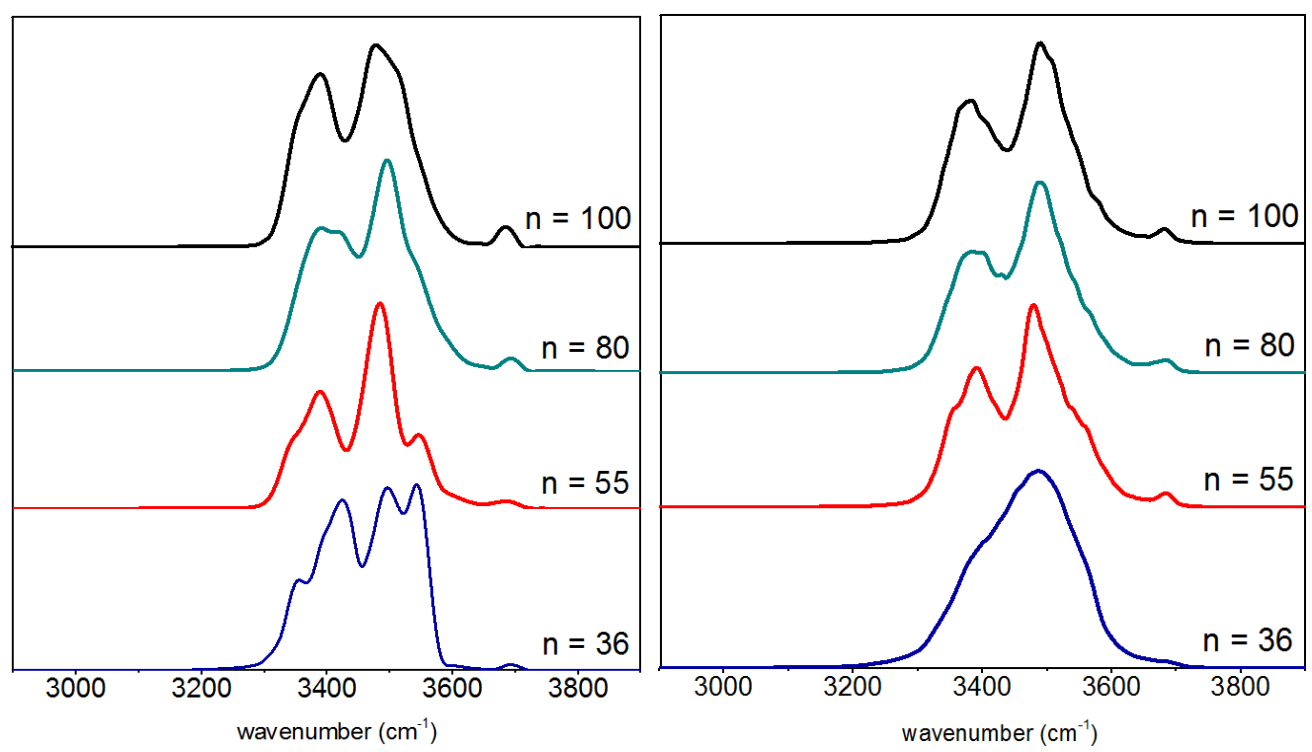

Figure 1: IR spectra for the sulfate in a water drop of increasing size (n from 36 to 100) at $100 \mathrm{~K}$ (left side) and $200 \mathrm{~K}$ (right side), using the AMOEBA14 water model.

IR spectra computed with the fluctuating charges model To enhance the flexibility of the water potential, the fluctuating charge model described in section 2.4 was applied to the same series of clusters to compute their IR spectra. The variation of the geometry was followed during the simulations. The average fluctuations increase up to $15 \%$ with the fluctuating charges model while they are a little more limited with the fixed charges model (Table 2). On average, the $\widehat{H O H}$ angle is smaller than its reference value with both models. The fluctuation of the oxygen and hydrogen charges allowed by the model is ca. $30 \%$. These geometry and charge fluctuations are in the same, physically reasonable, range than the ones observed by Xantheas with the TTM3-F water model ${ }^{35}$.

Table 2: Geometry fluctuations of the $\left[\mathrm{SO}_{4}\left(\mathrm{H}_{2} \mathrm{O}\right)_{80}\right]^{2-}$ cluster with respect to the reference data, $\Delta r$ for the $\mathrm{O}-\mathrm{H}$ bond, $\Delta \theta$ for the $\widehat{H O H}$ angle, between the AMOEBA14 and the fluctuating charges AMOEBA models, and fluctuations of the $\mathrm{O}$ and $\mathrm{H}$ charges.

\begin{tabular}{ccc}
\hline & Static charges & Fluctuating charges \\
\hline$\Delta r(\AA)$ & {$[-0.05:+0.1]$} & {$[-0.07:+0.13]$} \\
$\Delta \theta($ deg. & {$[-15:+10]$} & {$[-17:+13]$} \\
$\Delta \tilde{q}^{H}(\mathrm{e})$ & 0 & {$[-0.05:+0.06]$} \\
$\Delta \tilde{q}^{O}(\mathrm{e})$ & 0 & {$[-0.11:+0.11]$} \\
\hline
\end{tabular}


In Figure 2, the spectra for $n=36$ and $n=80$ are reported for both models in comparison with experiments. For the 36-molecule drop, the fluctuating charge model allows a broadening of the bonded $\mathrm{O}-\mathrm{H}$ band to reproduce accurately the lower frequency part until 3300 $\mathrm{cm}^{-1}$. For $\mathrm{n}=80$, the band is broader than the one computed with the fixed charge model, with a small red-shift for the high-frequency region. The double band shape is still present but attenuated. The free $\mathrm{O}-\mathrm{H}$ stretch band is at the same position with the same width with both models. Some intensity fluctuations are observed, mainly due to the sampling.
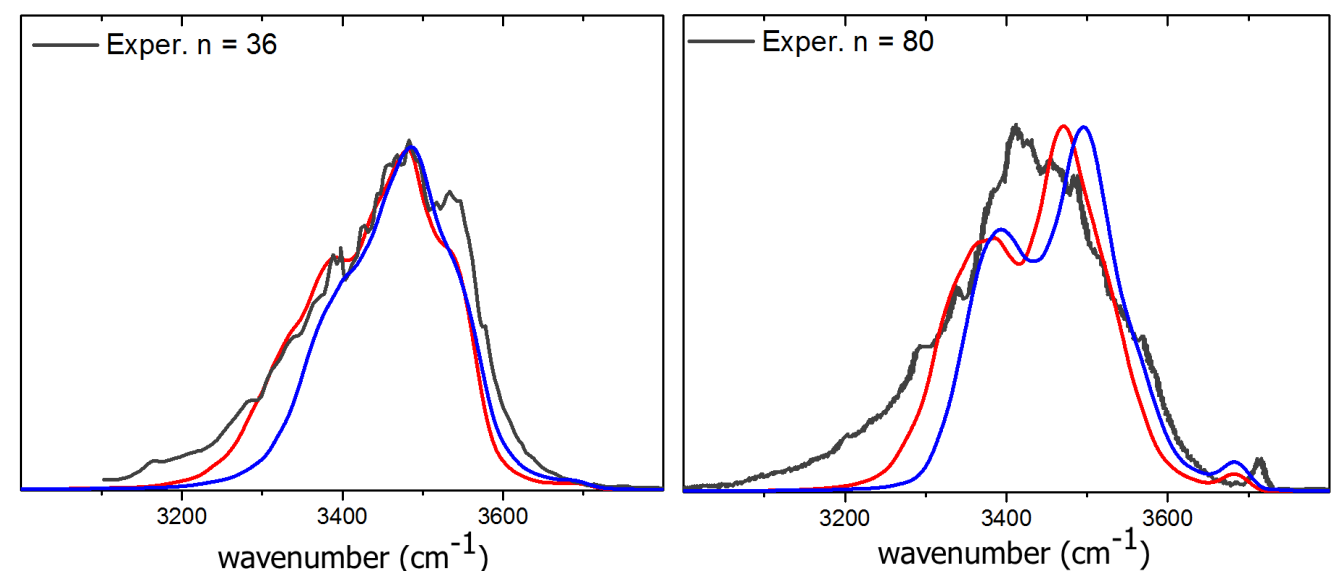

Figure 2: DACF-AMOEBA spectra at $200 \mathrm{~K}$ for $\left[\mathrm{SO}_{4}\left(\mathrm{H}_{2} \mathrm{O}\right)_{36}\right]^{2-}($ left $)$ and $\left[\mathrm{SO}_{4}\left(\mathrm{H}_{2} \mathrm{O}\right)_{80}\right]^{2-}$ (right) with both models with fixed charges (blue lines) and fluctuating charges (red lines). Experimental spectra at $130 \mathrm{~K}$ are represented in black from ref. ${ }^{11}$.

The DACF-AMOEBA spectra are reported in Figure 3 for the whole series, superimposed with the available experimental spectra ${ }^{11}$. These computed spectra have been averaged over ten MD simulations instead of three in Figure $\mathbf{2}$ for a total simulation time of 2 ns per spectrum. When comparing the global shape of the spectrum for $n=80$ between the two figures, the double-band of the bonded O-H region is strongly attenuated with a shoulder on the red part of the band. This highlights the crucial role of the sampling in the simulations in order to obtain IR spectra comparable to experiments. Looking at the series, a contribution below $3300 \mathrm{~cm}^{-1}$ is present with increasing number of molecules. It can be related to the emergence of a third and then a fourth solvation sphere leading to full AADD coordination 
of molecules in the first two spheres. Experimentally, the pure water cluster ${ }^{8}$ has a broader band than the hydrated sulfate one ${ }^{11,13}$ for the same number of water molecules. Consequently for the larger sizes, the influence of the sulfate is reduced but still significant with a long-range structural effect until 250 water molecules ${ }^{13}$. Furthermore, a progressive red-shift of the free $\mathrm{O}-\mathrm{H}$ band is observed with size increase due to a reduced influence of the ion.

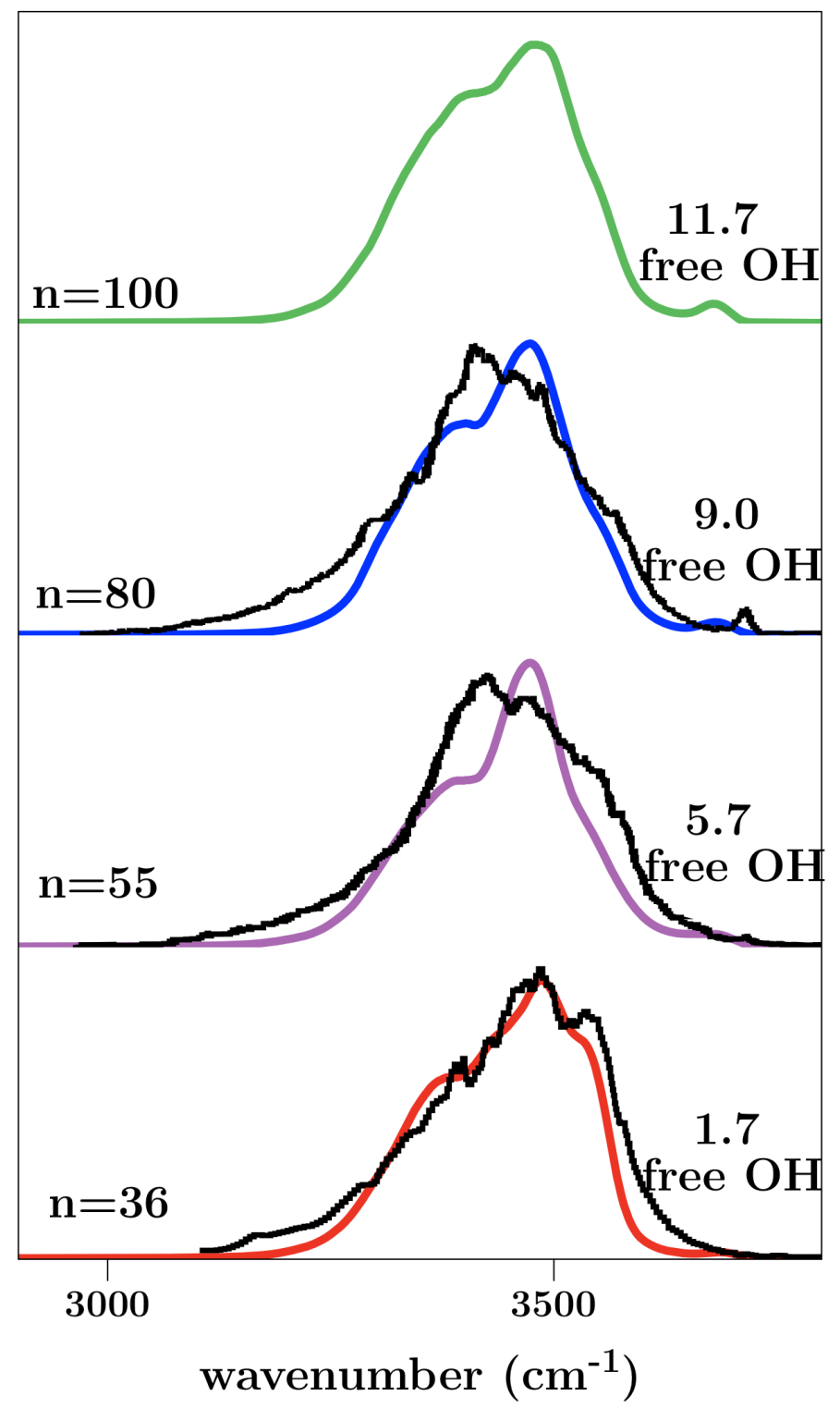

Figure 3: $\left[\mathrm{SO}_{4}\left(\mathrm{H}_{2} \mathrm{O}\right)_{n}\right]^{2-}$ spectra for $\mathrm{n}=36$ to 100 computed at $200 \mathrm{~K}$ using the AMOEBA fluctuating charge model in comparison with experimental spectra (black lines) ${ }^{11}$. The computed spectrum for $\mathrm{n}=55$ is superimposed with the IRPD spectrum for $\mathrm{n}=50$. 


\subsection{Mode assignments for $\left[\mathrm{SO}_{4}\left(\mathrm{H}_{2} \mathrm{O}\right)_{n}\right]^{2-}, \mathbf{n}=36$ and 80}

The DMD method can be used to assign vibrational modes by running short simulations. The scanning procedure described in section 2.3. provides access to the frequencies and mode assignments of a typical structure. Moreover, geometry fluctuations that occur during the DMD dynamics generate time changes of $\mathrm{OH}$ vibrators, yielding information about mode couplings. However, the method cannot provide an interpretation of the global spectrum obtained from a long MD simulation, e.g. 600 ps as typically used herein for computing DACF-AMOEBA spectra. To illustrate its capabilities, the DMD procedure was applied to a set of typical structures for drops with 36 and 80 water molecules.

\subsection{1 $\left[\mathrm{SO}_{4}\left(\mathrm{H}_{2} \mathrm{O}\right)_{36}\right]^{2-}$}

For the 36-molecule drop, three structures have been extracted from a MD simulation and optimized with AMOEBA (see Figure S1 in Supplementary Information). Structures are labeled N1/N2/N3/N4 where N1, N2, N3 and N4 stand for the numbers of water molecules in the first, second, third and fourth hydration shells, respectively. The most sable structure found by this limited search, W36-A, has a $12 / 18 / 6 / 0$ shell filling. W36-B $(+1 \mathrm{kcal} / \mathrm{mol})$ is of $11 / 18 / 6 / 1$ type and W36-C $(+2.9 \mathrm{kcal} / \mathrm{mol})$ is $11 / 18 / 7 / 0$. The latter two structures have been selected to account for some structural diversity.

While W36-A has no free O-H bond at its surface (in agreement with experiments, see reference $^{11}$ ), W36-B and $\mathrm{W} 36-\mathrm{C}$ have one free $\mathrm{OH}$ vibrator in a $\mathrm{AD}$ water molecule, with similar environments (donation to an AADD molecule, reception from an ADD molecule); comparison of these two cases will be taken as an illustrative example. For W36-C, this vibrator has a resonant absorption at $3726 \mathrm{~cm}^{-1}$, more blue-shifted than for AAD molecules because of the less constraining $\mathrm{AD}$ environment, the other vibrator is at $3448 \mathrm{~cm}^{-1}$. For W36- $\mathrm{B}$, the free $\mathrm{OH}$ vibrator is on resonance at $3663 \mathrm{~cm}^{-1}$ while the bonded $\mathrm{OH}$ absorbs energy at $3484 \mathrm{~cm}^{-1}$. To explain the differences, the time evolution of their $\mathrm{O}-\mathrm{H}$ distances is followed in Figure 4 at the free $\mathrm{OH}$ resonant absorption. The average distance for the 
bonded $\mathrm{OH}$ vibrator is larger in W36-C than in W36-B, because of a stronger hydrogen bond. Consequently, the reverse is observed for the free $\mathrm{OH}$ vibrators. In addition, while the free O-H elongation grows smoothly during excitation in W36-C, it does not in W36-B, especially during the first $100 \mathrm{fs}$. This is due to the stronger hydrogen bond in W36-B, leading to a smoother potential for the bonded O-H elongation and thus to strong oscillations, which in turn inhibit those of the free $\mathrm{OH}$ elongation. In all cases, the energy diffuses over time through bond couplings, so that both bonds vibrate significantly.
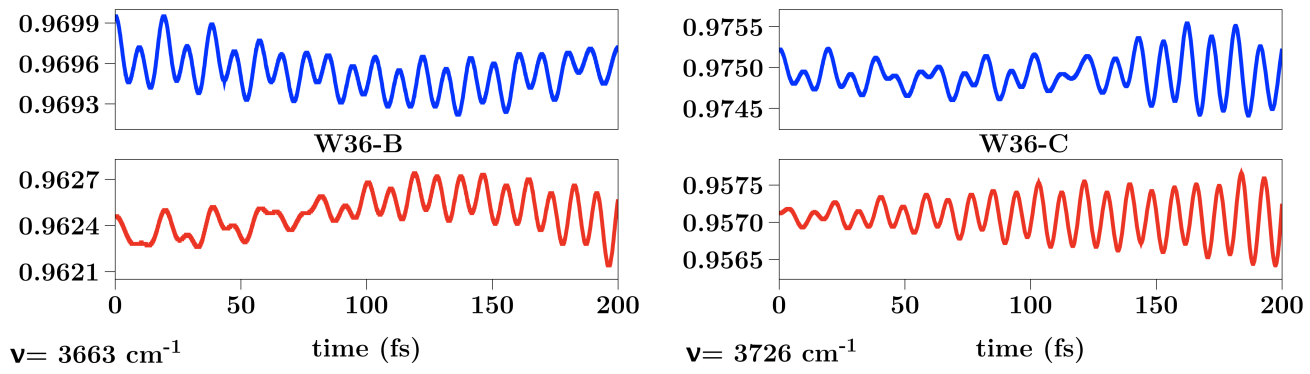

Figure 4: DMD simulations for $\left[\mathrm{SO}_{4}\left(\mathrm{H}_{2} \mathrm{O}\right)_{36}\right]^{2-}$ structures W36-B (left side) and W36-C (right side): time evolution of the $\mathrm{O}-\mathrm{H}$ distances (in $\AA$ ) for the water molecule with one free $\mathrm{O}-\mathrm{H}$, the free $\mathrm{OH}$ vibrator in red and, the bonded one in blue.

DMD assignments to hydration shells for the three structures are provided in Figure $\mathbf{5}$ and superimposed with the average DACF-AMOEBA spectrum. It should be noted that the DMD assignments only pertain to three individual structures, while the DACF simulations involve extensive sampling at $200 \mathrm{~K}$. In addition, bar heights are not absorption intensities, but are inversely proportional to the differences between the applied and the resonant response frequencies during the scan of the spectrum. Thus the superposition in Figure 5 only yields a tool to analyze the contributions of the hydration shells to the different regions of the spectrum. For the bonded $\mathrm{OH}$ region, a high density of frequencies is observed under the band maximum, i.e. $3450-3500 \mathrm{~cm}^{-1}$. They are attributed predominantly to molecules from the first and second shells. In the entire bonded OH massif, the DMD mode analysis reveals absorptions by $\mathrm{O}-\mathrm{H}$ bonds in all three hydration shells, because in this drop size range, significant diversity of environment exists (e.g. sulfate oxygens are not also 3-coordinated). 
At the low-frequency end of the massif, it is apparent from Figure 5 that most contributions arise from second shell molecules, which are also the most numerous. First shell absorptions in this area are found to arise from hydrogen bonds to second sphere waters as well as to sulfate oxygens.
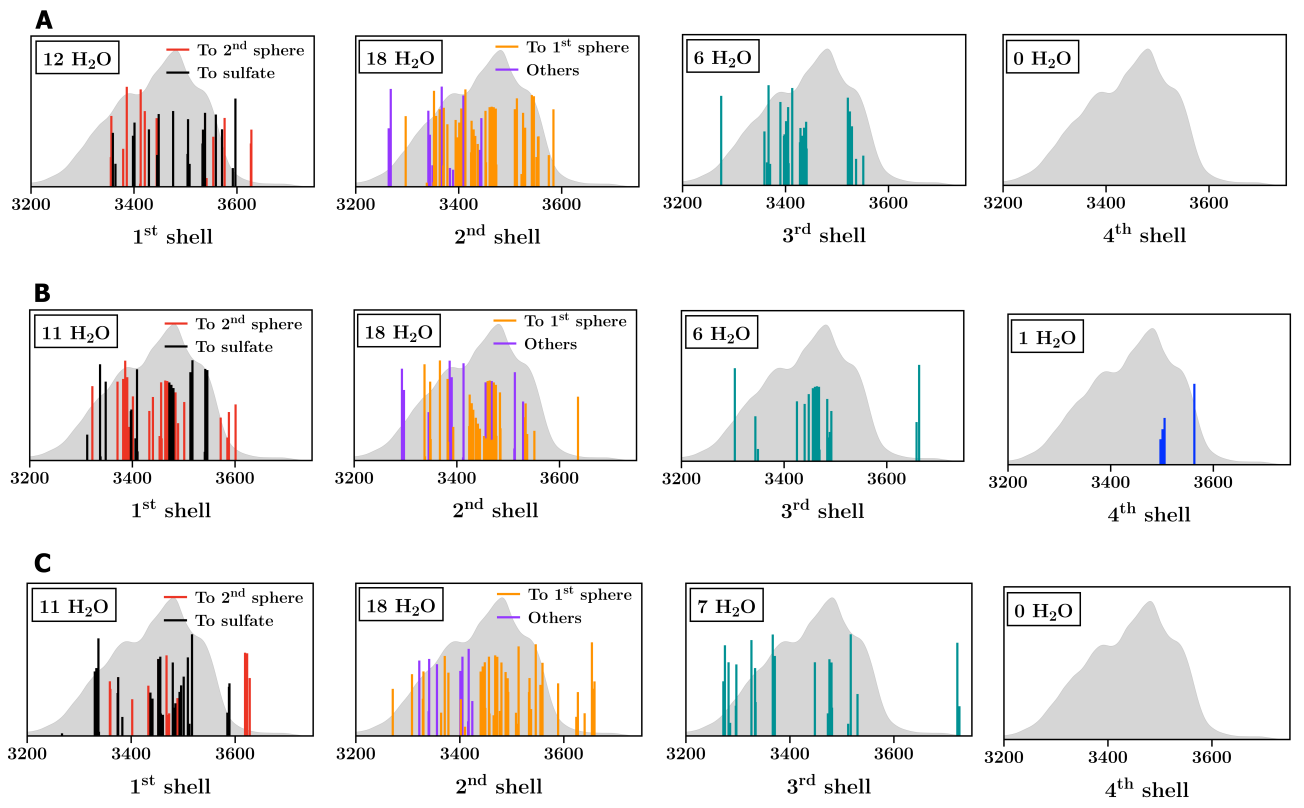

Figure 5: DMD attributions of the vibrational modes for the W36-A(12/18/6), W36$\mathrm{B}(11 / 18 / 6 / 1)$ and $\mathrm{W} 36-\mathrm{C}(11 / 18 / 7)$ structures of $\left[\mathrm{SO}_{4}\left(\mathrm{H}_{2} \mathrm{O}\right)_{36}\right]^{2-}$. DACF-AMOEBA spectrum at $200 \mathrm{~K}$ is plotted in grey.

\subsection{2 $\left[\mathrm{SO}_{4}\left(\mathrm{H}_{2} \mathrm{O}\right)_{80}\right]^{2-}$}

For the drop with 80 molecules, the two lowest-energy structures produced by the REMD simulation were selected and optimized before DMD assignments (Figure 6). The most stable structure W80-A is of 12/25/28/15 type, with six free O-H bonds at the cluster surface with vibrational signatures in the $3686-3708 \mathrm{~cm}^{-1}$ frequency range. W80-B is only $0.5 \mathrm{kcal} / \mathrm{mol}$ higher in energy than W80-A with 11/25/26/18 shell filling and five free O-H bonds in the $3672-3722 \mathrm{~cm}^{-1}$ frequency range.

The DMD analysis of both structures is provided in Figure 7 in order to get an overview of the attributions. Almost half of the molecules of the drop are in the first two hydration 


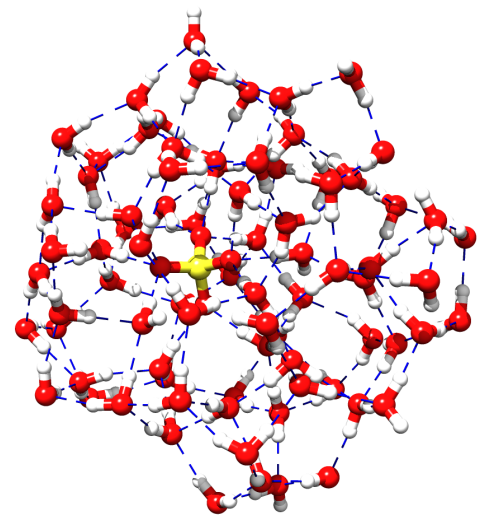

W80-A

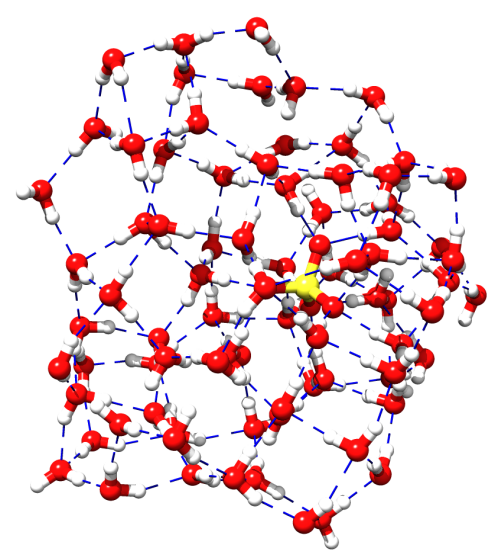

W80-B

Figure 6: Starting structures for DMD analyses for $\left[\mathrm{SO}_{4}\left(\mathrm{H}_{2} \mathrm{O}\right)_{80}\right]^{2-}$.

shells with relatively similar environments. As a result the vibrators of each molecule are generally coupled in symmetric and antisymmetric combinations at $\nu \leq 3350 \mathrm{~cm}^{-1}$ and $\nu$ $\geq 3425 \mathrm{~cm}^{-1}$, respectively. As expected, most of the bonded $\mathrm{O}-\mathrm{H}$ contributions arise from the first three shells, however strong hydrogen bonds also exist in the cluster surface as illustrated by strongly red-shifted bands from the fourth sphere. The free $\mathrm{O}-\mathrm{H}$ band arises from either $\mathrm{AAD}$ or $\mathrm{AD}$ molecules in both structures. In agreement with previously suggested assignments ${ }^{12}$, free $\mathrm{O}-\mathrm{H}$ frequencies are found to be larger in AAD molecules because they point toward the cluster exterior, while those in $\mathrm{AD}$ molecules are red-shifted because their orientations are less exterior, i. e. their dipoles interact slightly more strongly with the ion electric field.

Oscillations of several vibrators with strong resonance in the first two shells are analyzed by DMD and reported in Figure 8 . The vibrators involved are pointed out in Figure $\mathbf{7}$ with * symbols. (a) and (b) correspond to molecules in the first shell, (c) and (d) to molecules in the second shell. For (a), the distances of the two vibrators in the first shell molecule are initially similar. Their oscillation increases strongly with a clear antisymmetric coupling. For (b), the environment is similar to (a) however in this case the coupling is symmetric. The oscillation does not increase because intramolecular mode coupling is less favorable in a symmetric mode. The (c) situation is similar to (b) but for a molecule in the second 

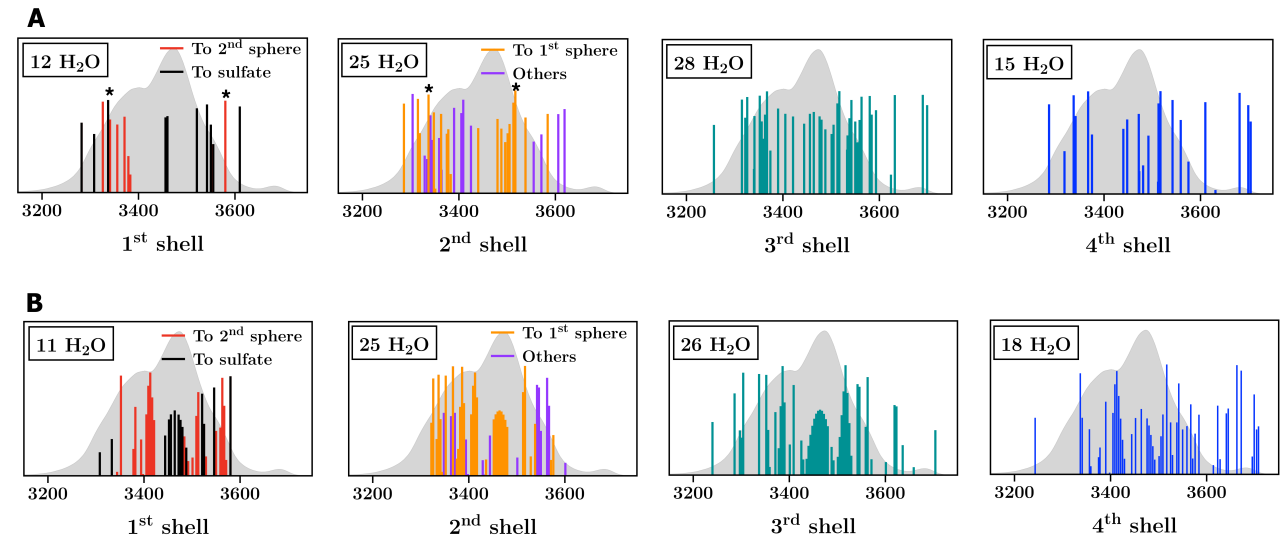

Figure 7: DMD attributions of the vibrational modes for the W80-A and W80-B structures of $\left[\mathrm{SO}_{4}\left(\mathrm{H}_{2} \mathrm{O}\right)_{80}\right]^{2-}$. DACF-AMOEBA spectrum at $200 \mathrm{~K}$ in grey.

shell and corresponds to a symmetric coupling with comparable environments for the two vibrators, one in interaction with a first shell molecule and the other with one in the second shell. However, the distances and amplitudes are in average larger than for (b). For (d), the molecule is in interaction with both one molecule in the first and one in the third shell with very different distances ( 1.78 vs $1.90 \AA$ ). It can be seen that a strong change in the environment occurs at ca. 100 fs. This is due to the flexibility of the third shell neighbour and it can be seen that the mode has evolved from a symmetric to an antisymmetric coupling of bond elongations.

An overall analysis of the bonded O-H massif indicates that for intermediate sizes $(\mathrm{n}=20$ 30) where the first hydration shell is not complete, very strong hydrogen bonds can be established from first to second shell molecules when the latter are of ADD type. Such O-H bonds in first shell waters undergo the largest red shifts of all. For growing cluster sizes (n from 30 to 80), most second shell molecules are of AADD types, making weaker individual hydrogen bonds. Thus the largest red shifts are no longer specific to interactions from the first to the second shells, they become spread out over both.

The DMD tool is found to be useful to explain the global shape of the spectrum and 

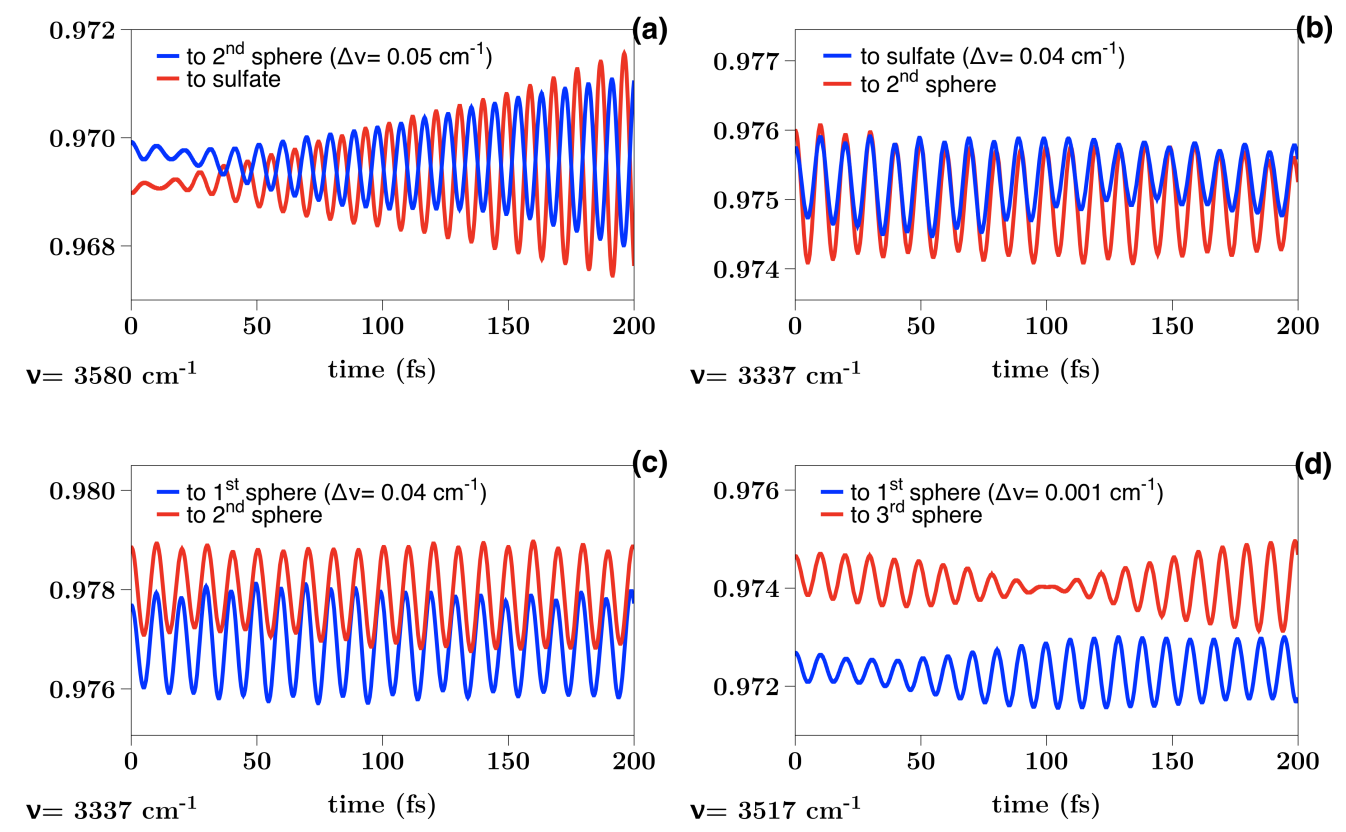

Figure 8: Time evolution of the $\mathrm{O}-\mathrm{H}$ distances for the water molecule in W80-A. DMD simulations for molecules in the first shell, (a) and (b), and molecules in the second shell, (c) and $(d)$. In each case, the indicated resonant absorption is for the mode that corresponds to the distance evolution in blue.

identify specific bands even if in some cases, a direct comparison with the DACF-AMOEBA spectrum may be misleading. This is the case in W80-B with multiple contributions of molecules in the fourth shell between 3410 and $3425 \mathrm{~cm}^{-1}$. These contributions do not come from several vibrators but from only one with a flexibility to enter in resonance on a large range. The frequency filter is a robust tool to allow the resonance on a variable range of frequencies but in such cases, intensities should be considered with caution.

\subsection{Ion influence on IR spectra}

In recent years, several groups have been able to produce gaseous size-selected nanodrops of hydrated ions containing dozens to hundreds of water molecules, and to record their IRPD spectra $^{7,9-23}$. The O-H stretch region in the $3000-3800 \mathrm{~cm}^{-1}$ range has revealed particularly useful because the stretching frequencies of $\mathrm{O}-\mathrm{H}$ bonds are very sensitive to their hydrogen bonding environment. In addition, this size range maintains a non-negligible fraction of 
surface relative to interior molecules, yielding access to signatures of all types of waters. IR spectra have been shown to be dependent upon both the ion charge and the drop size. They are generally made of a wide and intense feature corresponding to hydrogen-bonded O-H stretches, together with a much smaller and narrower band due to free O-H bonds at the cluster surface. The dependence of the position and relative intensity of the latter band to ion charge and drop size has been interpreted as the consequence of significant patterning of the hydrogen bond network by the ion, up to the third or fourth hydration shell at least ${ }^{12}$. The methods described above and their implementation as a complete suite make possible to model ionic drops in this size range, including their dynamics and vibrational spectra, with unprecedented accuracy. We have calculated DACF-AMOEBA spectra for a series of ions $\left[\mathrm{M}\left(\mathrm{H}_{2} \mathrm{O}\right)_{36}\right]^{x}, \mathrm{x}=-2$ to +3 , for which direct comparison to experiment ${ }^{12}$ is possible. Ions include $\mathrm{SO}_{4}^{2-}, \mathrm{I}^{-}, \mathrm{Br}^{-}, \mathrm{Cl}^{-}, \mathrm{F}^{-}, \mathrm{Cs}^{+}, \mathrm{K}^{+}, \mathrm{Na}^{+}, \mathrm{Li}^{+}, \mathrm{Ca}^{2+}$ and $\mathrm{La}^{3+}$. Analogous work was done for $\left[\mathrm{M}\left(\mathrm{H}_{2} \mathrm{O}\right)_{100}\right]^{x}, \mathrm{x}=-2$ to +3 with the same series of ions, as a demonstration that the method is straightforwardly applicable to larger systems. MD simulations were carried out at $200 \mathrm{~K}$, a temperature found to yield good match to the experimental profiles although their nominal temperature is $133 \mathrm{~K}$.

The entire $\mathrm{O}-\mathrm{H}$ stretch spectra computed for $\left[\mathrm{M}\left(\mathrm{H}_{2} \mathrm{O}\right)_{36}\right]^{x}, \mathrm{x}=-2$ to $+3, \mathrm{M}=\mathrm{SO}_{4}^{2-}$, $\mathrm{I}^{-}, \mathrm{Cs}^{+}, \mathrm{Li}^{+}, \mathrm{Ca}^{2+}$ and $\mathrm{La}^{3+}$ are shown in Figure 9. All computed spectra are shown in Figure S2. Free O-H frequencies maxima are listed in Table S3. It can be seen that the extended feature in the $3100-3650 \mathrm{~cm}^{-1}$ range, corresponding to bonded O-H stretches, is well reproduced qualitatively by simulations. Its shifting to the red with increasing ion charge (from -2 to +3 ) is also reproduced. Agreement is particularly good for sulfate and also for other anions, while the computed massif is somewhat over-shifted to the red for cations of increasing charge. This is likely due to the lack of charge transfer from the ion to first shell waters in the force field, which would reduce the charge density and thus the electric field at the cluster surface ${ }^{63}$. The strong $\mathrm{La}^{3+}$-water interactions also make this spectrum more 
dependent upon the starting structure used, which may require even more extensive DACF averaging.

The existence of a small and narrow band corresponding to free O-H stretches is also well reproduced, although it is red-shifted by $30-40 \mathrm{~cm}^{-1}$ relative to experiment. It is hardly present for sulfate in agreement with experiment ${ }^{11}$, however our simulations reveal an average number of free $\mathrm{O}-\mathrm{H}$ bonds of 1.7 (see Figure 3). This small ratio of $2.3 \%$ of free $\mathrm{O}-\mathrm{H}$ bonds may render their experimental detection particularly challenging. The peak maximum is red-shifted from $\mathrm{SO}_{4}^{2-}$ to $\mathrm{La}^{3+}$ by ca. $28 \mathrm{~cm}^{-1}$ experimentally ${ }^{12}$, a trend which is nicely reproduced by the DACF-AMOEBA spectra. Finally, the ratio of integrated free-OH band intensity to the total integrated band intensity is in excellent agreement with experiment for $\mathrm{I}^{-}(0.02)$ and its increase with increasing ion charge is also well reproduced, although with a smaller slope than for experiment since computed ratios are 0.05-0.06 instead of 0.06-0.10 for singly- to triply charged cations.

Analogous computations were carried out for $\left[\mathrm{M}\left(\mathrm{H}_{2} \mathrm{O}\right)_{100}\right]^{x}$, including 11 ions with $\mathrm{x}=$ -2 to +3 . Experiments are not systematically available for this size ${ }^{23}$, however trends can be compared to measurements for clusters with 245-255 water molecules ${ }^{13}$. As can be seen in Figure 10, there is much more relative intensity in the red part of the hydrogen-bonded massif $\left(3200-3350 \mathrm{~cm}^{-1}\right)$ than for $\mathrm{n}=36$, in agreement with the experimental trend. In addition, the width and frequency maxima of the computed IR spectra are much less dependent upon ion charge, both for the bound- and free-OH bands, than for the smaller clusters. This is consistent with a growing weight of water-water interactions relative to ion-water interactions when the cluster gets larger. Most molecules in the first two hydration shells are expected to have AADD bonding patterns, as well as some in the third shell. Thus, the dynamic continuum of hydrogen bond strengths becomes more similar to that in bulk water. As found for experimental bands, fitting the bonded-OH massif can be achieved using 


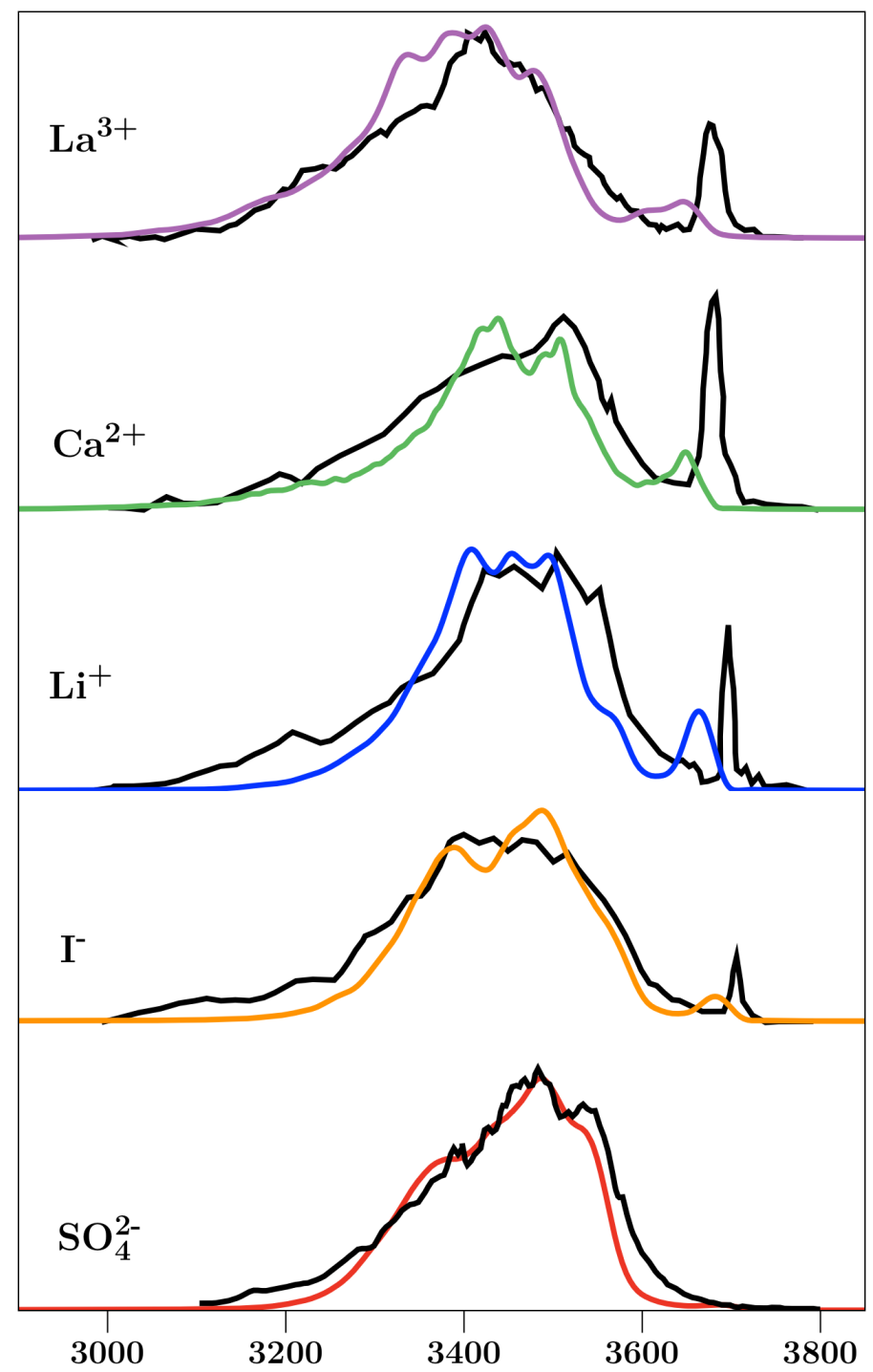

Figure 9: DACF-AMOEBA spectra at $200 \mathrm{~K}$ for $\left[M\left(\mathrm{H}_{2} \mathrm{O}\right)_{36}\right]^{x}, \mathrm{x}=-2$ to +3 , in the O-H stretching region superimposed with the experimental spectra in black ${ }^{9,11,12}$.

two gaussian functions, although the double maximum character is more pronounced than found experimentally. DMD assignments described above indicate however that this cannot be associated to two different types of hydrogen bonds or stretching motions.

The much smaller free $\mathrm{O}-\mathrm{H}$ stretching bands are computed at lower frequencies than found experimentally (by 30-40 $\mathrm{cm}^{-1}$ ), however they do show several trends in very good agreement with experiment. At a given drop size, their frequencies are found to diminish 
from $\mathrm{SO}_{4}^{2-}$ to $\mathrm{La}^{3+}$, however much less so for $\mathrm{n}=100$ (red shifting by $24 \mathrm{~cm}^{-1}$ ) than for $\mathrm{n}=36$ $\left(40 \mathrm{~cm}^{-1}\right)$. As discussed extensively by Williams et al. ${ }^{11-13,23}$, these trends are consistent with a major role played by Stark shifting of the O-H stretching at the drop surface by the ion-generated electric field. In addition, a blue shift is observed with growing size for cationcontaining drops, while the opposite is true for anion-containing ones. This is consistent with the opposite orientations of water $\mathrm{O}-\mathrm{H}$ bonds in the first hydration shell for opposite ion charges (towards the ion for anions, away from it for cations), an effect which carries its influence through hydrogen bonding, all the way to the drop surface ${ }^{23}$.

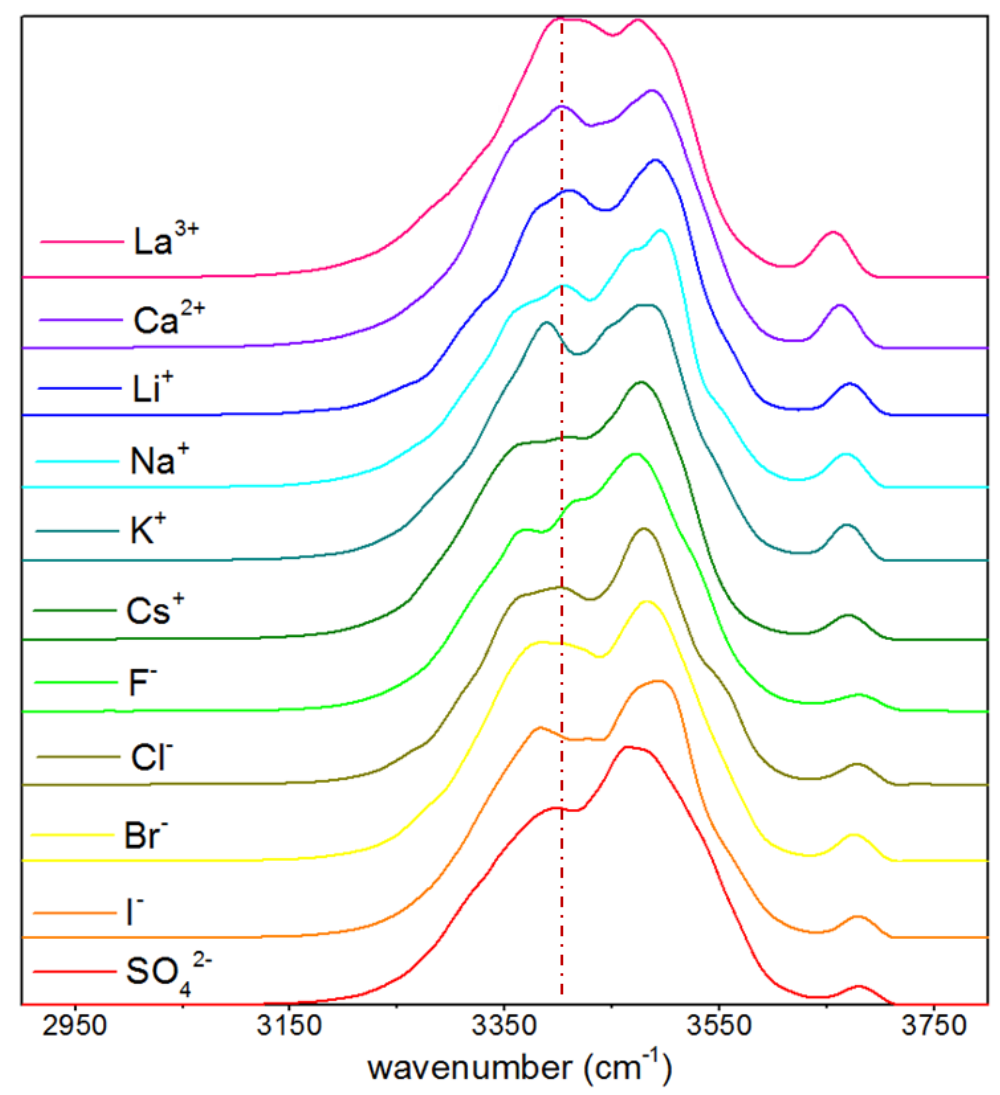

Figure 10: DACF-AMOEBA spectra at $200 \mathrm{~K}$ for $\left[M\left(\mathrm{H}_{2} \mathrm{O}\right)_{100}\right]^{x}, \mathrm{x}=-2$ to +3 , in the O-H stretching region. The dashed line represents the intensity maximum for pure bulk water. 


\section{Conclusions}

The O-H stretching signatures of ion-containing water drops have been investigated by molecular dynamics using the AMOEBA polarizable force field. Parameter adjustments for atomic multipoles and intramolecular force constants have been proposed to better reproduce gasphase spectroscopic data of water and sulfate-water drops. However, despite its refined electrostatic description coupled to an explicit polarization term, the AMOEBA model in its standard form is sometimes not flexible enough to catch the full spectroscopic response of complex and dynamic hydrogen bond network in these clusters. A fluctuating charge extension of the potential has been implemented to improve the computation of strongly anharmonic modes. Finite temperature spectra have been obtained for a series of sulfatewater drops with 36, 55, 80 and 100 water molecules. It has been shown that obtaining converged vibrational spectra requires more than three times 200 ps. The main features of the experimental spectra for the free and bonded O-H bands have been reproduced including their dependence upon drop size. Moreover, mode assignments have been made using the driven molecular dynamics tool with attributions to the $\mathrm{O}-\mathrm{H}$ vibrators in the various water shells around the sulfate anion, including couplings between modes. The DACF-AMOEBA method associated to the fluctuating charge model has also been used to compute IR spectra for 100-water drops containing various ions. The influence of the ion charge from -2 to +3 , and differences with the same bands between 36 and 100-water drops, have been found to match nicely the experimental trends, especially for the free $\mathrm{O}-\mathrm{H}$ stretching bands which have been shown previously to carry very useful structural information.

The improved AMOEBA model, implemented in computations of IR spectra from molecular dynamics simulations with the DACF method, together with the ability to assign bands with the DMD approach, provides a full package of simulation of IR spectra of highly flexible assemblies of large size. The agreement with experiments on IR spectra of drops of growing sizes opens the perspective of considering the transition from drops to bulk-like behavior in larger systems. 


\section{Supporting Information}

The Supporting Information is available free of charge on the ACS Publication website: DMD method details, hydration-shell definition, potential energies of 20-water clusters, vibrational frequencies of water monomer, free O-H frequency maxima for clusters with 36 and 100 water molecules, DACF-AMOEBA spectra at $200 \mathrm{~K}$ for the 11 ions in 36-molecule water drop.

\section{Acknowledgment}

CNRS and Ecole polytechnique are thanked for funding. F.T. and C.J. thank Ecole Polytechnique for a PhD fellowship.

\section{References}

(1) Zhou, Y.; Morais-Cabral, J. H.; Kaufman, A.; MacKinnon, R. Chemistry of ion coordination and hydration revealed by a $\mathrm{K}^{+}$channel-Fab complex at $2.0 \AA$ resolution. Nature 2001, 414, 43-48.

(2) Rasband, M. N. Ion Channels and Excitable Cells. Nature Education 2010, 3, 41.

(3) Richards, L. A.; Schafer, A. I.; Richards, B. S.; Corry, B. Quantifying barriers to monovalent anion transport in narrow non-polar pores. Phys. Chem. Chem. Phys. 2012, $14,11633-11638$.

(4) Sahu, S.; Di Ventra, M.; Zwolak, M. Dehydration as a Universal Mechanism for Ion Selectivity in Graphene and Other Atomically Thin Pores. Nano Letters 2017, 17, $4719-4724$.

(5) Finlayson-Pitts, B. J.; Pitts-Jr., J. N. Chemistry of the Upper and Lower Atmosphere - Theory, Experiments and Applications; Academic Press: San Diego, 2000. 
(6) Kirkby, J.; Duplissy, J.; Sengupta, K.; Frege, C.; Gordon, H.; Williamson, C.; Heinritzi, M.; Simon, M.; Yan, C.; Almeida, J. et al. Ion-induced nucleation of pure biogenic particles. Nature 2016, 533, 521-526.

(7) Walters, R. S.; Pillai, E. D.; Duncan, M. A. Solvation Dynamics in $\mathrm{Ni}^{+}\left(\mathrm{H}_{2} \mathrm{O}\right)_{n}$ Clusters Probed with Infrared Spectroscopy. J. Am. Chem. Soc. 2005, 127, 16599-16610.

(8) Pradzynski, C. C.; Forck, R. M.; Zeuch, T.; Slavicek, P.; Buck, U. A fully size-resolved perspective on the crystallization of water clusters. Science 2012, 337, 1529-1532.

(9) Bush, M. F.; Saykally, R. J.; Williams, E. R. Infrared Action Spectra of $\mathrm{Ca}^{2+}\left(\mathrm{H}_{2} \mathrm{O}\right)_{11-69}$ Exhibit Spectral Signatures for Condensed-Phase Structures with Increasing Cluster Size. J. Am. Chem. Soc. 2008, 130, 15482-15489.

(10) Bush, M. F.; O’Brien, J. T.; Prell, J. S.; Wu, C.-C.; Saykally, R. J.; Williams, E. R. Hydration of Alkaline Earth Metal Dications: Effects of Metal Ion Size Determined Using Infrared Action Spectroscopy. J. Am. Chem. Soc. 2009, 131, 13270-13277.

(11) O'Brien, J. T.; Prell, J. S.; Bush, M. F.; Williams, E. R. Sulfate Ion Patterns Water at Long Distance. J. Am. Chem. Soc. 2010, 132, 8248-8249.

(12) Prell, J. S.; O'Brien, J. T.; Williams, E. R. Structural and Electric Field Effects of Ions in Aqueous Nanodrops. J. Am. Chem. Soc. 2011, 133, 4810-4818.

(13) O'Brien, J. T.; Williams, E. R. Effects of Ions on Hydrogen-Bonding Water Networks in Large Aqueous Nanodrops. J. Am. Chem. Soc. 2012, 134, 10228-10236.

(14) Nagornova, N. S.; Rizzo, T. R.; Boyarkin, O. V. Interplay of Intra- and Intermolecular H-Bonding in a Progressively Solvated Macrocyclic Peptide. Science 2012, 336, 320.

(15) Mizuse, K.; Fujii, A. Infrared spectroscopy of large protonated water clusters $\mathrm{H}^{+}\left(\mathrm{H}_{2} \mathrm{O}\right)_{20-50}$ cooled by inert gas attachment. Chem. Phys. 2013, 419, $2-7$. 
(16) Cooper, R. J.; Chang, T. M.; Williams, E. R. Hydrated Alkali Metal Ions: Spectroscopic Evidence for Clathrates. J. Phys. Chem. A 2013, 117, 6571-6579.

(17) DiTucci, M. J.; Heiles, S.; Williams, E. R. Role of Water in Stabilizing Ferricyanide Trianion and Ion-Induced Effects to the Hydrogen-Bonding Water Network at Long Distance. J. Am. Chem. Soc. 2015, 137, 1650-1657.

(18) Fournier, J. A.; Wolke, C. T.; Johnson, C. J.; Johnson, M. A.; Heine, N.; Gewinner, S.; Schöllkopf, W.; Esser, T. K.; Fagiani, M. R.; Knorke, H. et al. Site-specific vibrational spectral signatures of water molecules in the magic $\mathrm{H}_{3} \mathrm{O}^{+}\left(\mathrm{H}_{2} \mathrm{O}\right)_{20}$ and $\mathrm{Cs}^{+}\left(\mathrm{H}_{2} \mathrm{O}\right)_{20}$ clusters. Proc. Nat. Acad. Sci. USA 2014, 111, 18132-18137.

(19) Fournier, J. A.; Wolke, C. T.; Johnson, M. A.; Odbadrakh, T. T.; Jordan, K. D.; Kathmann, S. M.; Xantheas, S. S. Snapshots of Proton Accommodation at a Microscopic Water Surface: Understanding the Vibrational Spectral Signatures of the Charge Defect in Cryogenically Cooled $\mathrm{H}^{+}\left(\mathrm{H}_{2} \mathrm{O}\right)_{n=2-28}$ Clusters. J. Phys. Chem. A 2015, 119, 9425-9440.

(20) Heiles, S.; Cooper, R. J.; DiTucci, M. J.; Williams, E. R. Hydration of guanidinium depends on its local environment. Chem. Sci. 2015, 6, 3420-3429.

(21) Marsh, B. M.; Voss, J. M.; Garand, E. A dual cryogenic ion trap spectrometer for the formation and characterization of solvated ionic clusters. J. Chem. Phys. 2015, 143, 204201.

(22) Cooper, R. J.; DiTucci, M. J.; Chang, T. M.; Williams, E. R. Delayed Onset of Crystallinity in Ion-Containing Aqueous Nanodrops. J. Am. Chem. Soc. 2016, 138, 96-99.

(23) Cooper, R. J.; O’Brien, J. T.; Chang, T. M.; Williams, E. R. Structural and electrostatic effects at the surfaces of size- and charge-selected aqueous nanodrops. Chem. Sci. 2017, 8, 5201-5213. 
(24) Griffin, G. B.; Young, R. M.; Ehrler, O. T.; Neumark, D. M. Electronic relaxation dynamics in large anionic water clusters: $\left(\mathrm{H}_{2} \mathrm{O}\right)_{n}$ and $\left(\mathrm{D}_{2} \mathrm{O}\right)_{n}(\mathrm{n}=25-200)$. J. Chem. Phys. 2009, 131, 194302.

(25) Servage, K. A.; Silveira, J. A.; Fort, K. L.; Russell, D. H. Evolution of Hydrogen-Bond Networks in Protonated Water Clusters $\mathrm{H}^{+}\left(\mathrm{H}_{2} \mathrm{O}\right)_{n}(\mathrm{n}=1$ to 120) Studied by Cryogenic Ion Mobility-Mass Spectrometry. J. Phys. Chem. Lett. 2014, 5, 1825-1830.

(26) Servage, K. A.; Fort, K. L.; Silveira, J. A.; Shi, L.; Clemmer, D. E.; Russell, D. H. Unfolding of Hydrated Alkyl Diammonium Cations Revealed by Cryogenic Ion MobilityMass Spectrometry. J. Am. Chem. Soc. 2015, 137, 8916-8919.

(27) Dang, L. X.; Rice, J. E.; Caldwell, J.; Kollman, P. A. Ion solvation in polarizable water: molecular dynamics simulations. J. Am. Chem. Soc. 1991, 113, 2481-2486.

(28) Jungwirth, P.; Tobias, D. J. Molecular Structure of Salt Solutions: A New View of the Interface with Implications for Heterogeneous Atmospheric Chemistry. J. Phys. Chem. B 2001, 105, 10468-10472.

(29) Grossfield, A.; Ren, P.; Ponder, J. W. Ion Solvation Thermodynamics from Simulation with a Polarizable Force Field. J. Am. Chem. Soc. 2003, 125, 15671-15682.

(30) Brown, E. C.; Mucha, M.; Jungwirth, P.; Tobias, D. J. Structure and Vibrational Spectroscopy of Salt Water/Air Interfaces: Predictions from Classical Molecular Dynamics Simulations. J. Phys. Chem. B 2005, 109, 7934-7940.

(31) Chang, T.-M.; Dang, L. X. Recent Advances in Molecular Simulations of Ion Solvation at Liquid Interfaces. Chem. Rev. 2006, 106, 1305-1322.

(32) Duvail, M.; Villard, A.; Nguyen, T.-N.; Dufrêche, J.-F. Thermodynamics of Associated Electrolytes in Water: Molecular Dynamics Simulations of Sulfate Solutions. J. Phys. Chem. B 2015, 119, 11184-11195. 
(33) Thaunay, F.; Ohanessian, G.; Clavaguéra, C. Dynamics of ions in a water drop using the AMOEBA polarizable force field. Chem. Phys. Lett. 2017, 671, 131-137.

(34) Burnham, C. J.; Xantheas, S. S. Development of transferable interaction models for water. IV. A flexible, all-atom polarizable potential (TTM2-F) based on geometry dependent charges derived from an ab initio monomer dipole moment surface. J. Chem. Phys. 2002, 116, 5115.

(35) Fanourgakis, G. S.; Xantheas, S. S. Development of transferable interaction potentials for water. V. Extension of the flexible, polarizable, Thole-type model potential (TTM3F, v. 3.0) to describe the vibrational spectra of water clusters and liquid water. J. Chem. Phys. 2008, 128, 074506.

(36) Ren, P.; Ponder, J. W. Polarizable atomic multipole water model for molecular mechanics simulation. J. Phys. Chem. B 2003, 107, 5933-5947.

(37) Ponder, J. W.; Wu, C.; Ren, P.; Pande, V. S.; Chodera, J. D.; Schnieders, M. J.; Haque, I.; Mobley, D. L.; Lambrecht, D. S.; DiStasio, R. A. et al. Current Status of the AMOEBA Polarizable Force Field. J. Phys. Chem. B 2010, 114, 2549-2564.

(38) Semrouni, D.; Sharma, A.; Dognon, J.-P.; Ohanessian, G.; Clavaguéra, C. Finite Temperature Infrared Spectra from Polarizable Molecular Dynamics Simulations. J. Chem. Theory Comput. 2014, 10, 3190-3199.

(39) Thaunay, F.; Dognon, J.-P.; Ohanessian, G.; Clavaguera, C. Vibrational mode assignment of finite temperature infrared spectra using the AMOEBA polarizable force field. Phys. Chem. Chem. Phys. 2015, 17, 25968-25977.

(40) Deng, S.; Wang, Q.; Ren, P. Estimating and modeling charge transfer from the SAPT induction energy. J. Comput. Chem. 2017, 26, 2222-2231. 
(41) Piquemal, J.-P.; Perera, L.; Cisneros, G. A.; Ren, P.; Pedersen, L. G.; Darden, T. A. Towards accurate solvation dynamics of divalent cations in water using the polarizable AMOEBA force field: From energetics to structure. J. Chem. Phys. 2006, 125, 054511.

(42) Wu, J. C.; Piquemal, J.-P.; Chaudret, R.; Reinhardt, P.; Ren, P. Polarizable Molecular Dynamics Simulation of $\mathrm{Zn}(\mathrm{II})$ in Water Using the AMOEBA Force Field. J. Chem. Theo. Comput. 2010, 6, 2059-2070.

(43) Semrouni, D.; Isley, W. C.; Clavaguéra, C.; Dognon, J.-P.; Cramer, C. J.; Gagliardi, L. Ab Initio Extension of the AMOEBA Polarizable Force Field to $\mathrm{Fe}^{2+}$. J. Chem. Theo. Comput. 2013, 9, 3062-3071.

(44) Kumar, M.; Simonson, T.; Ohanessian, G.; Clavaguéra, C. Structure and Thermodynamics of Mg:Phosphate Interactions in Water: A Simulation Study. ChemPhysChem 2015, 16, 658-665.

(45) Marjolin, A.; Gourlaouen, C.; Clavaguéra, C.; Ren, P. Y.; Wu, J. C.; Gresh, N.; Dognon, J.-P.; Piquemal, J.-P. Toward accurate solvation dynamics of lanthanides and actinides in water using polarizable force fields: from gas-phase energetics to hydration free energies. Theor. Chem. Acc. 2012, 131, 1198.

(46) Ren, P.; Ponder, J. W. Consistent treatment of inter- and intramolecular polarization in molecular mechanics calculations. J. Comput. Chem. 2002, 23, 1497-1506.

(47) Halgren, T. A. The representation of van der Waals (vdW) interactions in molecular mechanics force fields: potential form, combination rules, and vdW parameters. J. Am. Chem. Soc. 1992, 114, 7827-7843.

(48) Allinger, N. L.; Yuh, Y. H.; Lii, J. H. Molecular mechanics. The MM3 force field for hydrocarbons. 1. J. Am. Chem. Soc. 1989, 111, 8551-8566. 
(49) Lambrecht, D. S.; Clark, G. N. I.; Head-Gordon, T.; Head-Gordon, M. Exploring the Rich Energy Landscape of Sulfate-Water Clusters $\mathrm{SO}_{4}^{2-}-\left(\mathrm{H}_{2} \mathrm{O}\right)_{n=3-7}$ : An Electronic Structure Approach. J. Phys. Chem. A 2011, 115, 11438-11454.

(50) Zhou, J.; Santambrogio, G.; Brümmer, M.; Moore, D. T.; Wöste, L.; Meijer, G.; Neumark, D. M.; Asmis, K. R. Infrared spectroscopy of hydrated sulfate dianions. J. Chem. Phys. 2006, 125, 111102.

(51) Stone, A. J. Distributed multipole analysis, or how to describe a molecular charge distribution. Chem. Phys. Lett. 1981, 83, 233-239.

(52) Stone, A. J.; Alderton, M. Distributed multipole analysis Methods and applications. Mol. Phys. 1985, 56, 1047-1064.

(53) Laury, M. L.; Wang, L.-P.; Pande, V. S.; Head-Gordon, T.; Ponder, J. W. Revised Parameters for the AMOEBA Polarizable Atomic Multipole Water Model. J. Phys. Chem. B 2015, 119, 9423-9437.

(54) Ponder, J. W. TINKER - Software Tools for Molecular Design (version 6), http://dasher.wustl.edu/tinker. 2014.

(55) Nosé, S. A unified formulation of the constant temperature molecular dynamics methods. J. Chem. Phys. 1984, 81, 511-519.

(56) Bowman, J. M.; Zhang, X.; Brown, A. Normal-mode analysis without the Hessian: A driven molecular-dynamics approach. J. Chem. Phys. 2003, 119, 646-650.

(57) Kaledin, M.; Brown, A.; Kaledin, A. L.; Bowman, J. M. Normal mode analysis using the driven molecular dynamics method. II. An application to biological macromolecules. $J$. Chem. Phys. 2004, 121, 5646-5653.

(58) Brown, A.; Kaledin, M.; Bowman, J.; Kaledin, A. Normal Mode Analysis; Chapman and Hall/CRC, 2005; pp 281-300. 
(59) Bentwood, R.; Barnes, A.; Orville-Thomas, W. Studies of intermolecular interactions by matrix isolation vibrational spectroscopy: Self-association of water. J. Mol. Spectrosc. 1980, $84,391-404$.

(60) Page, R. H.; Frey, J. G.; Shen, Y.-R.; Lee, Y.-T. Infrared predissociation spectra of water dimer in a supersonic molecular beam. Chem. Phys. Lett. 1984, 106, 373-376.

(61) Xantheas, S. S.; Dunning, T. H. Ab initio studies of cyclic water clusters $\left(\mathrm{H}_{2} \mathrm{O}\right)_{n}$, n=1-6. I. Optimal structures and vibrational spectra. J. Chem. Phys. 1993, 99, 8774.

(62) Wang, L.-P.; Head-Gordon, T.; Ponder, J. W.; Ren, P.; Chodera, J. D.; Eastman, P. K.; Martinez, T. J.; Pande, V. S. Systematic Improvement of a Classical Molecular Model of Water. J. Phys. Chem. B 2013, 117, 9956-9972.

(63) Clavaguéra, C.; Calvo, F.; Dognon, J.-P. Theoretical study of the hydrated Gd ${ }^{3+}$ ion: Structure, dynamics, and charge transfer. J. Chem. Phys. 2006, 124, 074505. 
TOC Graphic

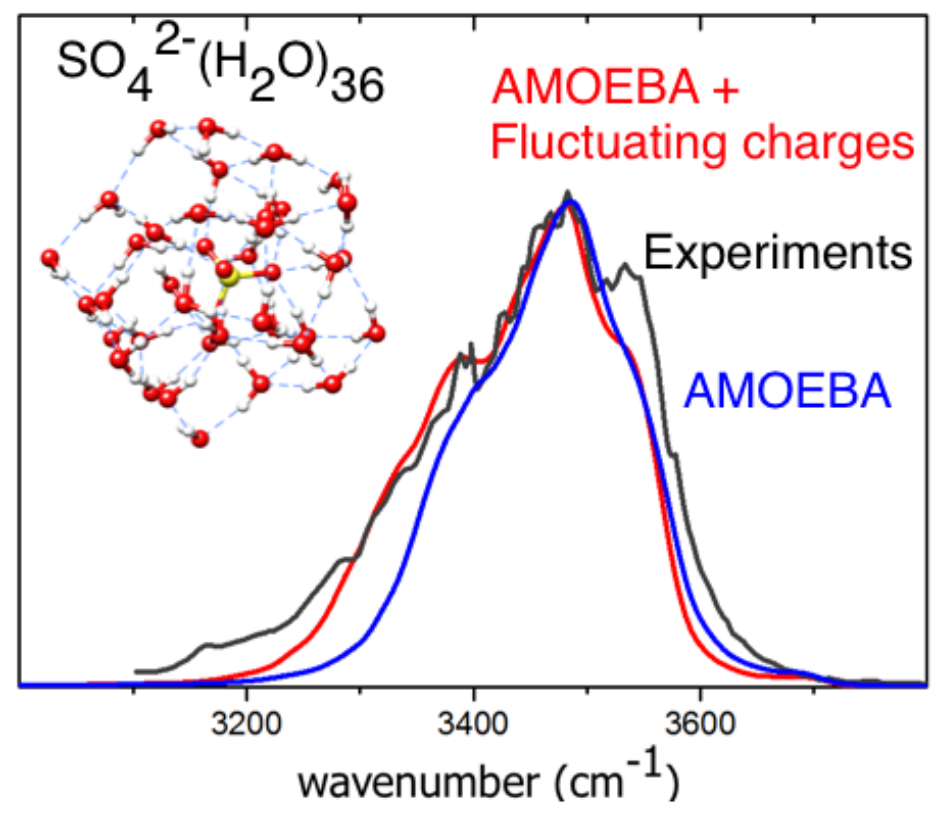

